\title{
Plasmids are vectors for redundant chromosomal genes in the Bacillus cereus group
}

\author{
Jinshui Zheng, Ziyu Guan, Shiyun Cao, Donghai Peng, Lifang Ruan, Daohong Jiang and Ming Sun*
}

\begin{abstract}
Background: Prokaryotic plasmids have played significant roles in the evolution of bacterial genomes and have a great impact on the metabolic functions of the host cell. Many bacterial strains contain multiple plasmids, but the relationships between bacterial plasmids and chromosomes are unclear. We focused on plasmids from the Bacillus cereus group because most strains contain several plasmids.

Results: We collected the genome sequences of 104 plasmids and 20 chromosomes from B. cereus group strains, and we studied the relationships between plasmids and chromosomes by focusing on the pan-genomes of these plasmids and chromosomes. In terms of basic features (base composition and codon usage), the genes on plasmids were more similar to the chromosomal variable genes (distributed genes and unique genes) than to the chromosomal core genes. Although all the functional categories of the chromosomal genes were exhibited by the plasmid genes, the proportions of each category differed between these two gene sets. The 598 gene families shared between chromosomes and plasmids displayed a uniform distribution between the two groups. A phylogenetic analysis of the shared genes, including the chromosomal core gene set, indicated that gene exchange events between plasmids and chromosomes occurred frequently during the evolutionary histories of the strains and species in this group. Moreover, the shared genes between plasmids and chromosomes usually had different promoter and terminator sequences, suggesting that they are regulated by different elements at the transcriptional level.
\end{abstract}

Conclusions: We speculate that for the entire B. cereus group, adaptive genes are preserved on both plasmids and chromosomes; however, in a single cell, homologous genes on plasmids and the chromosome are controlled by different regulators to reduce the burden of maintaining redundant genes.

Keywords: Plasmid, Chromosome, Pan-genome, Bacillus cereus group

\section{Background}

Horizontal gene transfer (HGT) plays an important role in bacterial evolution by providing foreign genetic material for gene exchange between prokaryotes [1]. One of the most important contributors to HGT is plasmids, which can be transferred between cells as vectors for genes and can provide a basis for genomic rearrangements via homologous recombination [2]. In this process, events in which genes are gained and/or lost force bacterial genomes to evolve. Moreover, many adaptive genes contained by plasmids are transferred, and these genes play important roles in bacterial adaptation to changing environments $[3,4]$.

\footnotetext{
* Correspondence: m98sun@mail.hzau.edu.cn

State Key Laboratory of Agricultural Microbiology, Huazhong Agricultural University, Wuhan 430070, China
}

(C) 2015 Zheng et al.; licensee Biomed Central. This is an Open Access article distributed under the terms of the Creative Commons Attribution License (http://creativecommons.org/licenses/by/4.0), which permits unrestricted use, distribution, and reproduction in any medium, provided the original work is properly credited. The Creative Commons Public Domain Dedication waiver (http://creativecommons.org/publicdomain/zero/1.0/) applies to the data made available in this article unless otherwise stated.

Plasmids have been studied for different purposes by many researchers. These studies have mainly focused on the intrinsic characteristics and accessory functions of plasmids. Among the former topics, plasmid replication, maintenance and mobilization have been the major subjects [5,6]; among the latter, contributions to antibiotic resistance (AR) and virulence have been the primary concerns [7]. Recently, as increasing numbers of plasmid genomic sequences have become available, systematic analyses of the dynamics and relationships among plasmids and their contributions to bacterial genomic evolution have become feasible. Tamminen et al. used network methods to study all of the 2,343 plasmids with available genomic sequences and described these plasmids' evolutionary dynamics and interrelationships [8]. By analyzing the plasmids of genus Acinetobacter, the same research group found that although most of these plasmids lack 
mobilization and transfer functions, they likely have a long history of rearrangements with other plasmids and with chromosomes [9]. Moreover, other research has revealed that plasmids have played more important roles than viruses in the evolution of bacterial genomes [10]. In addition to mediating HGT among different bacterial cells, plasmids contribute to bacterial evolution via their role in the formation and propagation of operons, a process in which plasmids have been likened to scribbling pads [11].

Because plasmids coexist with chromosomes in bacterial cells, the relationships between plasmids and chromosomes are critical for understanding the evolution and diversity of bacterial genomes. These relationships have been directly studied by focusing on gene exchange events between plasmids and chromosomes. Such events can be caused by transposons, phages, integrons and plasmids [12-15]. In addition, transposons and integrons can be found on both plasmids and chromosomes, and phages can be integrated into chromosomes and plasmids as prophages [16-18]. Even plasmids have been found to frequently integrate into chromosomes as integrative and conjugative elements [19]. However, no systematic analysis has closely examined the relationships between plasmids and chromosomes on a genome-wide scale. For example, in a particular species, how do plasmids affect chromosomal structures, what is the frequency of genetic exchange events between plasmids and chromosomes, and why are some genes harbored by both plasmids and chromosomes? In a previous study, we used the Bacillus cereus group as a model to explore the evolution and dynamics of plasmids [20]. In the present study, we use the $B$. cereus group as a model to study the relationships between plasmids and chromosomes by focusing on the genes that are shared between them.

Members of the $B$. cereus group are found in diverse environments, including soil, water, and animal hosts, and they include species of $B$. anthracis, B. cereus, $B$. thuringiensis and four more variable species, B. cytotoxicus, B. mycoides, B. pseudomycoides, and B. weihenstephanensis $[21,22]$. Plasmids are important for defining the first three species $[21,23,24]$. The plasmids in this group display strain-dependent distribution, with some strains containing no plasmids, whereas others have many (more than 10) [25-27]. Some of these plasmids have small genome sizes, only $2 \mathrm{~kb}$ [28], whereas others are very large, up to $600 \mathrm{~kb}$. Even within the same cell, the genome sizes of different plasmids vary widely; for example, B. thuringiensis CT-43 has 10 plasmids with genome sizes ranging from $6 \mathrm{~kb}$ to $300 \mathrm{~kb}$ [26]. In our recent work, we found that megaplasmids larger than $100 \mathrm{~kb}$ may have originated from integration events of smaller plasmids [20]. Furthermore, as reported previously, the total amount of plasmid DNA in a single $B$. thuringiensis cell is greater than that of chromosomal DNA [29]. This finding raises a question:
What is the nature of the relationship between plasmids and the chromosome?

We studied the relationships between chromosomes and plasmids by focusing on their shared genes. Clusters of orthologous groups (COGs) and base composition analyses indicated that plasmids may contain an additional copy of a variable chromosomal region. We also examined genetic exchanges between plasmids and chromosomes by focusing on the basic features of their shared genes.

\section{Results}

\section{Plasmids of the $B$. cereus group share dynamic gene pools with chromosomes}

We focused on pan-genomic plasmids and chromosomes to study the relationships between plasmids and chromosomes. The numbers of MCL (Markov Cluster) family members obtained using the OrthoMCL tool for the chromosomal core gene set (genes shared by all of the 20 chromosomes), chromosomal distributed gene set (genes shared by more than one chromosome but less than 20), chromosomal unique gene set (all the individual genes present on only one chromosome), plasmid distributed gene set (genes shared by more than one plasmid) and plasmid unique gene set (all the individual genes present on only one plasmid) were 2009, 3933, 6813, 1121 and 4934 , respectively. There were no core genes shared by all the plasmids. Overall, there were 598 gene families shared by plasmids and chromosomes.

We compared the basic features of genes from plasmids and chromosomes by analyzing the base composition of the gene sets described above. The average GC content of the genes on plasmids (34.1\%) was more similar to that the two types of variable genes $(34.9 \%$ for chromosomal unique genes and $34.5 \%$ for chromosomal distributed genes) than to that of the chromosomal core genes (37.1\%) $\left(\mathrm{P}=0.48,0.32\right.$ and $2.3 \times 10^{-6}$, Mann-Whitney test) (Figure 1A). A codon usage analysis with CAI (codon adaptation index) indicated that the plasmid genes showed no difference from the chromosomal variable genes (the $\mathrm{P}$ values for the plasmid genes compared with the two types of variable genes were 0.05 and 0.55 , respectively; Mann-Whitney test) but were significantly different from the chromosomal core genes $\left(\mathrm{P}<2.2 \times 10^{-16}\right.$, Mann-Whitney test) (Figure 1B). This finding indicates that the genes on plasmids share similar features with the variable genes (distributed genes and unique genes) of chromosomes, and the plasmids and chromosomes share the same dynamic gene pool.

We focused on functions determined by plasmids and found that all of the COG categories represented by chromosomes could be found on plasmids (Figure 2). By computing the proportion of gene families for each COG functional category, we found that approximately one-third of all the COG categories showed similar 


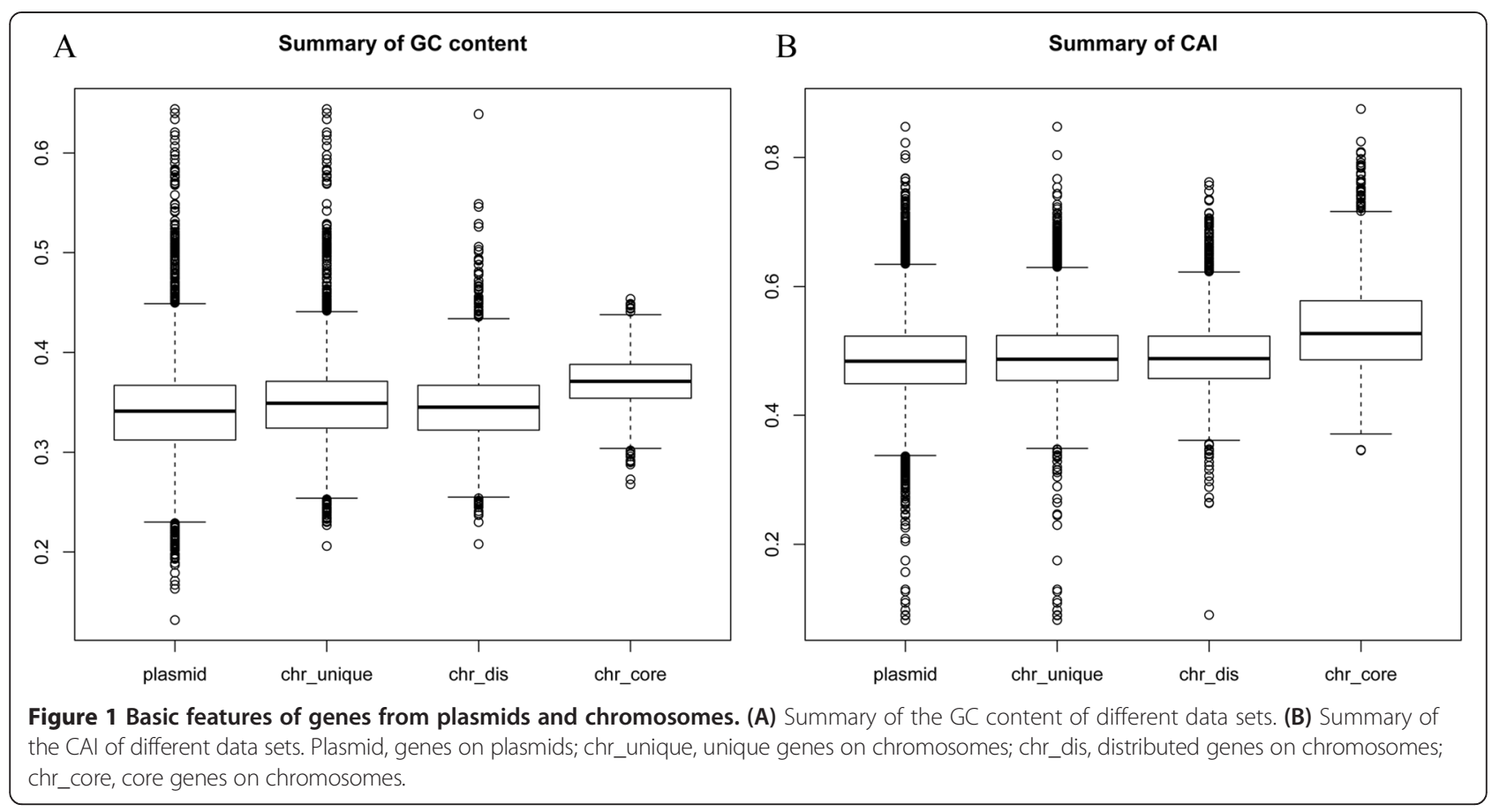

distributions on plasmids and chromosomes. The other two-thirds of the COG categories exhibited different distribution characteristics between plasmids and chromosomes. Gene families involved in replication, recombination, and repair represented the largest proportion of plasmid genes, but they occupied a significantly smaller proportion of the chromosomes $\left(\mathrm{P}<2.2 \times 10^{-16}\right.$, onesided binomial test). Moreover, gene families involved in

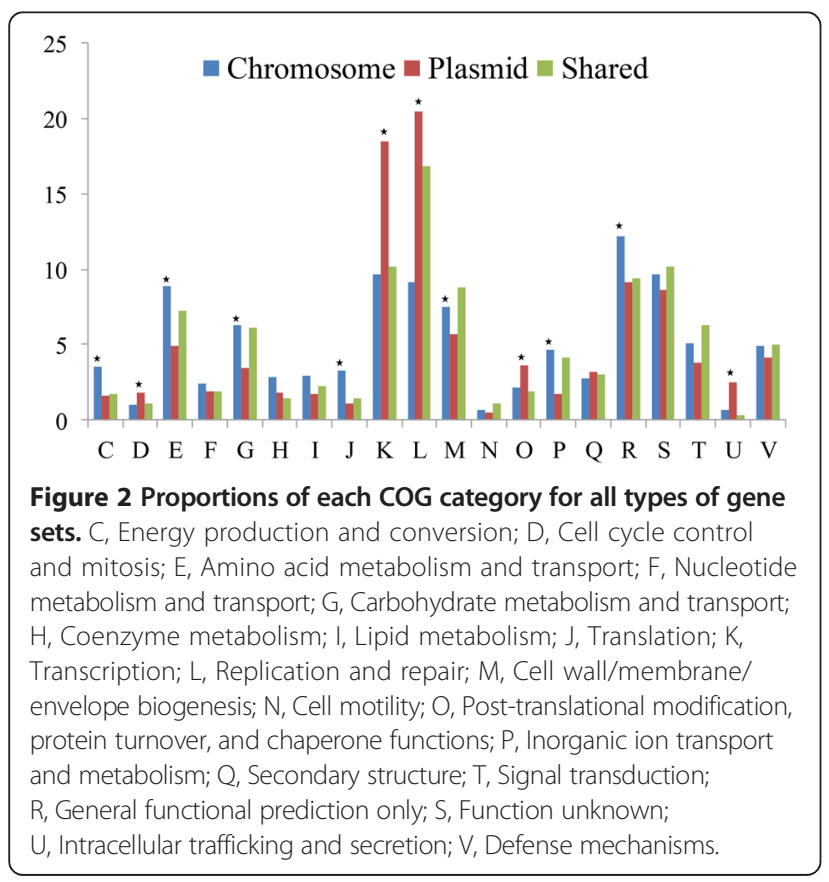

transcription were also significantly more enriched on plasmids than on chromosomes $\left(\mathrm{P}<2.2 \times 10^{-16}\right)$. On plasmids, these two types of gene families constituted almost half of the total gene families with known COG annotations. Other gene families, such as those involved in posttranslational modifications, protein turnover, chaperoning $\left(\mathrm{P}=2.83 \times 10^{-5}\right)$ and intracellular trafficking, secretion, and vesicular transport $\left(\mathrm{P}=1.12 \times 10^{-13}\right)$, were also enriched on plasmids. Conversely, the proportion of gene families involved in basal metabolism, such as those involved in amino acid transport and metabolism $\left(\mathrm{P}=6.08 \times 10^{-11}\right)$, carbohydrate transport and metabolism, lipid metabolism $\left(\mathrm{P}=3.34 \times 10^{-8}\right)$, inorganic ion transport and metabolism $\left(\mathrm{P}=1.24 \times 10^{-11}\right)$ and energy production and conversion $\left(\mathrm{P}=1.77 \times 10^{-6}\right)$ was significantly lower on plasmids than on chromosomes. In addition, gene families involved in translation were significantly more frequently found on chromosomes than on plasmids $\left(\mathrm{P}=1.37 \times 10^{-9}\right)$.

\section{Genetic exchange events between plasmids and} chromosomes have occurred frequently during the evolutionary history of the $B$. cereus group

Shared genes (homologous DNA fragments) between plasmids and chromosomes are the result of genetic exchange events. The 598 gene families shared by plasmids and chromosomes were found to be distributed across all the categories of the chromosomal gene set. For chromosomes, the largest number of shared genes was found in the distributed gene set, which included 342 gene families. The second largest number was in the unique gene set, which had 216 families, and the remainder was in the core 
gene set (Figure 3A). Equal numbers of shared genes from the distributed set were shared by one or multiple plasmids (Figure 3B), indicating that both single and multiple genetic exchange events among plasmids and chromosomes occurred during evolutionary history. Two-thirds of the shared genes from the chromosomal unique set were shared by one plasmid (Figure 3C); these may have resulted from recent genetic exchanges.

Genetic exchange events between chromosomes and plasmids were not restricted to certain regions; we found a uniform distribution of these shared genes throughout the chromosome. For example, the distribution of shared genes on the chromosome of $B$. anthracis Ames Ancestor showed no significant difference from a uniform random distribution ( $\mathrm{P}=0.34$, Mann-Whitney test). The same result was observed for plasmids, which generally exhibited uniformly located shared genes (for pBMB171, $\mathrm{P}=0.42$, Mann-Whitney test).

The numbers of shared genes between a given plasmid and different chromosomes varied greatly. We found that among the 20 genomes studied, the plasmid/ chromosome pairs with the greatest number of shared genes were never in the same cell. The most extreme example was the plasmid pBWB401 from a B. weihenstephanensis strain. This plasmid shared fewer than 50 genes with most of the $B$. cereus group chromosomes, but it shared 93 genes with B. cereus B4264. In fact, this plasmid and chromosome pair shared a DNA fragment of $105 \mathrm{~kb}$ (base pairs 3,422,398-3,528,167 of the B. cereus B4264 chromosome), including 57 coding sequences, with an average nucleotide sequence identity greater than $95 \%$. A recent genetic exchange may have occurred between pBWB401 and the B. cereus chromosome, after which the plasmid and the chromosome were separated.

Many genetic exchange events involved multiple genes (Additional file 1: Table S3). When the 57 uninterrupted genes shared by plasmid pBWB401 and chromosome $B$. cereus B4264 were excluded, 155 (29\%) of the 541 genes shared by plasmids and chromosomes constituted 58 operons. The smallest operon consisted of 2 genes, and the largest contained 9 genes. The genes in the same operon exhibited functional relatedness.
Genetic exchange events occurred frequently during the evolutionary histories of the members of the B. cereus group. Of the 40 shared genes of the chromosomal core gene set, 19 were exchanged between chromosomes and plasmids during the formation of the species; these 19 genes appeared as outgroups to the chromosomal homologous genes on the phylogenetic trees. For 13 of these 19 genes, the exchange events occurred only on plasmids after the different species' lineages had formed; there was no evidence of recent homologous recombination with chromosomal genes (see example in Figure 4A). The other 6 genes were frequently exchanged between plasmids and chromosomes, and some duplication of genes on chromosomes was caused by these events (see example in Figure 4B). Among the 11 shared genes that were exchanged by plasmids after the formation of $B$. cereus group lineages, some were from lineage I or II (see example in Figure 4C) and others on different plasmids had different sources (see example in Figure 4D).

When the COG functions of the shared genes were analyzed, we found that the genes that were most frequently shared among different plasmids were those that function as transporters. As shown in Table 1, among the 55 shared genes with known COG annotations in the chromosomal core and extended core gene sets (genes shared by more than 19 chromosomes), 16 were annotated as transporters. Additionally, among the 18 genes with known COG annotations that were shared by more than 10 plasmids, 7 were transporter-associated genes. The gene that was shared by the most chromosomes and plasmids was a transporter-associated gene encoding the substrate-binding component of an $\mathrm{ABC}$ type oligopeptide import system containing type 2 periplasmic binding folds. Other shared genes frequently contained in the chromosomal core and extended core sets were annotated as enzymes that participate in carbon and nitrogen metabolism. Among the 216 shared genes from the chromosomal unique set, the predominant functions were related to recombination; 17 and 7 genes were predicted to encode transposases and resolvases, respectively.

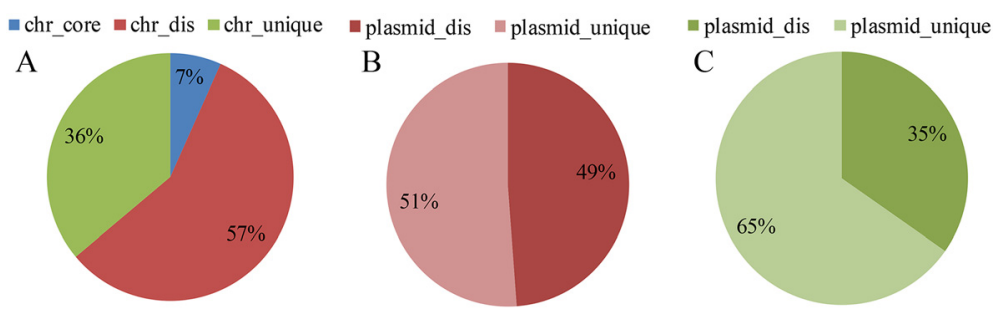

Figure 3 The 598 gene families shared by plasmids and chromosomes were distributed over all the categories of the chromosomal gene set. Each of the three gene sets contained shared genes (A). Shared genes from the distributed set were shared by one and more than one plasmid equally (B). Two-thirds of the shared genes from the chromosomal unique set were shared by one plasmid (C). 


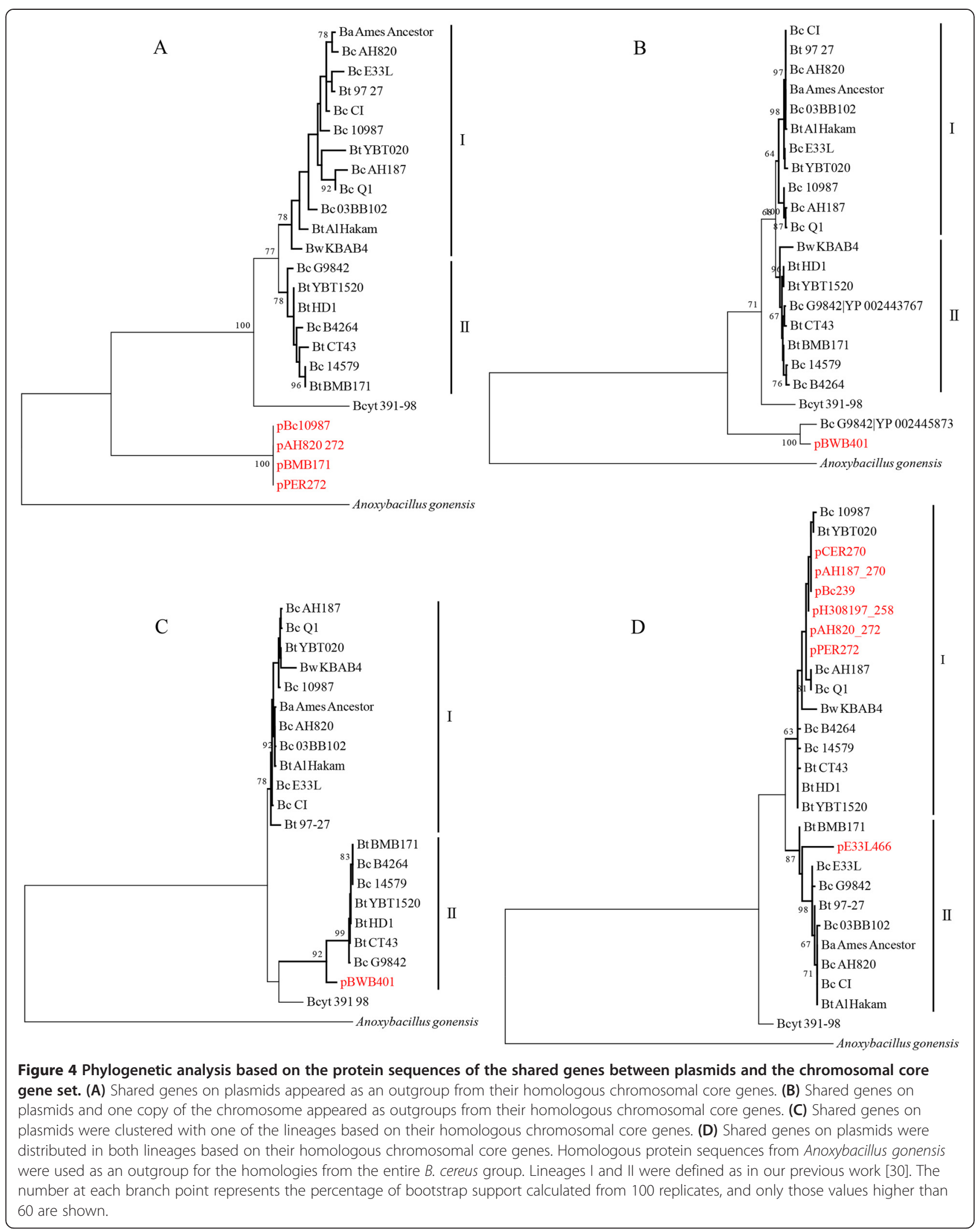


Table 1 COG annotations of the shared genes among the chromosomal core and extended core sets

\begin{tabular}{|c|c|c|c|}
\hline COG ID & Functional annotation & Chromosome number & Plasmid number \\
\hline 32477 & Predicted membrane protein [Function unknown] & 20 & 7 \\
\hline 131886 & Stage $V$ sporulation protein $\mathrm{AE}$ & 20 & 6 \\
\hline 131885 & Stage $\mathrm{V}$ sporulation protein $\mathrm{AC}$ & 20 & 6 \\
\hline 183504 & Stage $V$ sporulation protein $A D$ & 20 & 6 \\
\hline 178955 & ATP-dependent Clp protease proteolytic subunit & 20 & 5 \\
\hline 105987 & Hypothetical protein & 20 & 5 \\
\hline 193180 & MacB-like periplasmic core domain. & 20 & 4 \\
\hline 73014 & $\begin{array}{l}\text { This family is composed of MJ0796 ATP-binding cassette, macrolide-specific } \\
\text { ABC-type efflux carrier (MacAB), and proteins involved in cell division (FtsE) } \\
\text { and release of lipoproteins from the cytoplasmic membrane (LolCDE) }\end{array}$ & 20 & 4 \\
\hline 162057 & Arsenic-resistance protein & 20 & 4 \\
\hline 31088 & $\begin{array}{l}\text { Response regulators consisting of a CheY-like receiver domain and a } \\
\text { winged-helix DNA-binding domain }\end{array}$ & 20 & 4 \\
\hline 162505 & RND family efflux transporter, MFP subunit & 20 & 4 \\
\hline 115457 & Sugar transport protein & 20 & 4 \\
\hline 184117 & Arsenate reductase & 20 & 4 \\
\hline 181585 & Glucose-1-dehydrogenase & 20 & 4 \\
\hline 32652 & Zn-ribbon-containing protein involved in phosphonate metabolism & 20 & 3 \\
\hline 163006 & Polysaccharide deacetylase family sporulation protein PdaB & 20 & 3 \\
\hline 31331 & ABC-type antimicrobial peptide transport system, ATPase component & 20 & 3 \\
\hline 31475 & Uncharacterized conserved protein & 20 & 3 \\
\hline 197627 & Methyl-accepting chemotaxis-like domains (chemotaxis sensory transducer) & 20 & 3 \\
\hline 190390 & FtsX-like permease family & 20 & 3 \\
\hline 179411 & Adenine phosphoribosyltransferase & 20 & 2 \\
\hline 31326 & ABC-type multidrug transport system, ATPase component & 20 & 2 \\
\hline 32452 & Sugar phosphate permease & 20 & 1 \\
\hline 151609 & Protein of unknown function & 20 & 1 \\
\hline 188197 & Penicillin-binding protein, 1A family & 20 & 1 \\
\hline 34374 & Predicted membrane protein & 20 & 1 \\
\hline 31911 & Predicted transcriptional regulators & 20 & 1 \\
\hline 189896 & Formate/nitrite transporter & 20 & 1 \\
\hline 34876 & $\begin{array}{l}\text { Uncharacterized protein involved in cytokinesis, contains TGc } \\
\text { (transglutaminase/protease-like) domain }\end{array}$ & 20 & 1 \\
\hline 30931 & Uncharacterized membrane-associated protein & 20 & 1 \\
\hline 191813 & Major facilitator superfamily & 20 & 1 \\
\hline 162221 & Cysteine synthase $A$ & 20 & 1 \\
\hline 181811 & Membrane-bound transcriptional regulator LytR & 20 & 1 \\
\hline 31361 & $\begin{array}{l}\text { Transcriptional regulators containing a DNA-binding HTH domain and an } \\
\text { aminotransferase domain (MocR family) and their eukaryotic orthologs }\end{array}$ & 20 & 1 \\
\hline 179521 & D-serine dehydratase & 20 & 1 \\
\hline 188607 & D-alanyl-lipoteichoic acid biosynthesis protein DltD & 20 & 1 \\
\hline 162128 & Carboxylate/amino acid/amine transporter & 20 & 1 \\
\hline 110729 & Collagenase & 20 & 1 \\
\hline 129987 & Amino acid transporter & 20 & 1 \\
\hline 173869 & $\begin{array}{l}\text { The substrate-binding component of an ABC-type oligopeptide } \\
\text { import system containing the type } 2 \text { periplasmic binding fold }\end{array}$ & 19 & 13 \\
\hline
\end{tabular}


Table 1 COG annotations of the shared genes among the chromosomal core and extended core sets (Continued)

\begin{tabular}{llll}
\hline 163059 & Germination protein, Ger(x)C family & 19 & 7 \\
183898 & N-acetylglucosamine-binding protein A & 19 & 6 \\
189798 & Sodium/hydrogen exchanger family & 19 & 6 \\
30836 & Putative regulatory ligand-binding protein related to C-terminal domains of K channels & 19 & 19 \\
178836 & L-lactate dehydrogenase & 19 & 1 \\
31520 & Transcriptional regulators, similar to M & 19 & 1 \\
31856 & Acetyltransferases, including N-acetylases of ribosomal proteins & 19 & 1 \\
48387 & Nitroreductase-like family 4 & 19 & 1 \\
176695 & C-terminal domain of Sphingobium chlorophenolicum & 19 & 1 \\
145290 & 2,6-dichloro-p-hydroquinone 1,2-dioxygenase (PcpA) and similar proteins & 19 & 1 \\
110729 & BCCT family transporter & 19 & 1 \\
30749 & Predicted esterase & 19 & 1 \\
31331 & ABC-type antimicrobial peptide transport system, ATPase component & 19 & 1 \\
147640 & NosL. NosL is one of the accessory proteins of the nos (nitrous oxide reductase) gene cluster & 19 & 1 \\
162053 & Serine transporter & 19 & 1 \\
\hline
\end{tabular}

Shared genes between plasmids and chromosomes are regulated by different elements

Although many genes had been exchanged between plasmids and chromosomes, most of them had different promoters and terminators. We focused on genes shared between plasmids and the chromosome from the same host. The promoter and terminator sequences of a gene are located upstream and downstream of the coding sequence. We compared the upstream and downstream sequences of each of the 419 pairs of shared genes from the same host and found that only 139 genes had similar upstream and downstream sequences. Among the other 280 gene pairs, 240 had different upstream sequences, meaning these gene pairs had different promoters; 246 had different downstream sequences, meaning that these gene pairs had different terminators; and 206 pairs had different upstream and downstream sequences, indicating different promoters and terminators (Figure 5). This finding suggests that approximately two-thirds of the shared genes between plasmids and the chromosome from the same host are controlled at the transcriptional level by different elements.

This suggestion is supported by the reported transcriptome data for B. thuringiensis serovar chinensis CT-43 [31]. We examined this strain and focused on the 12 shared gene pairs composed of only one gene on the chromosome and one on a plasmid. We found that the shared genes with similar upstream and downstream sequences usually had similar transcriptional dynamics among the four time points, representing mid-exponential growth phase, early-stationary growth phase, midstationary growth phase and the time point when $30 \%$ of mother cells are lysed, whereas those with different upstream and/or downstream sequences had different transcriptional dynamics (Additional file 2: Table S4). The CT43_CH0952/CT43_P281096 gene pair, which had similar upstream and downstream sequences, had similar transcriptional dynamics during the four time points. Other shared gene pairs had different upstream and/or downstream sequences, and the genes in these pairs differed in their transcriptional dynamics.

\section{Discussion}

Many strains of the $B$. cereus group contain several plasmids with different replicons, and these plasmids have different genome sizes, ranging from 2 to $600 \mathrm{~kb}$. However,

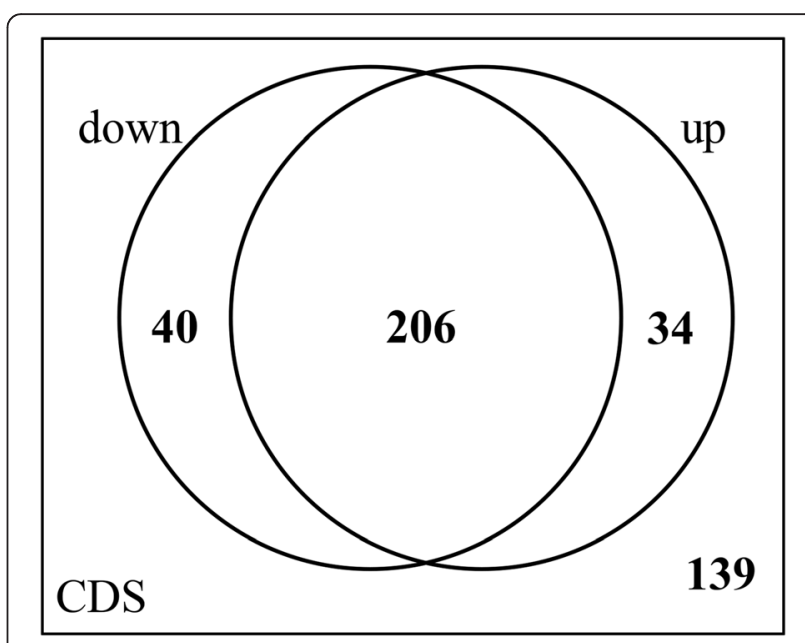

Figure 5 Among the $\mathbf{4 1 9}$ gene pairs from the same host, only 139 genes had similar upstream and downstream sequences. CDS, coding sequence of shared gene pairs; up, upstream 200-bp sequences; down, downstream 100-bp sequences. 
the functions determined by these plasmids have rarely been considered; only those with apparent pathogenic features have been well studied, such as the function of the two plasmids of $B$. anthracis that contribute to anthrax disease [21], the function of the emetic B. cereus plasmids that determine the emetic syndrome and the functions of some $B$. thuringiensis plasmids that cause toxicity to insects $[21,24]$. The other plasmids have not been well studied, although they represent the majority of the $B$. cereus group plasmids. This study focused on the pan-genome of the entire group.

We considered all of the plasmids as a group. Genes contained by plasmids were found to be similar to chromosomal variable genes but different from chromosomal core genes in terms of the basic features and the functions they provide. Genes on plasmids and chromosomal variable genes were considered non-essential genes, and they only differed in their location. However, genes on plasmids showed some differences from chromosomal variable genes; the former were enriched in functions of transcriptional regulation, but the latter were not (Additional file 3: Figure S1). This finding indicates that plasmids show some differences from chromosomes in the functions of the genes they harbor. However, all of the functional categories determined by the chromosomal core genes were presented by genes on plasmids. This finding indicates that genes with functions in basic metabolism and even some genes with essential functions for bacterial survival were also present on plasmids. This phenomenon has also been reported in other bacteria: nearly $11 \%$ of the genes on plasmid p42e of Rhizobium etli CFN42 participate in primary metabolism [32]. We predict that for the entire group, many of the genes that function in basic metabolism are present as two copies, one on the chromosome and the other on a plasmid. However, some essential genes were never found on plasmids within this group, such as genes for different types of ribosomal RNA; this differentiated the plasmids from the chromosomes.

Genetic exchange events have occurred frequently between plasmids and chromosomes, and almost all the regions of the chromosome were affected by these events. Moreover, because some of these events occurred along with the formation of the species, we predict that some plasmids of this group have long histories and were obtained by their hosts prior to lineage formation. During the long course of evolutionary history, many genes were harbored on both plasmids and chromosomes. For the entire group or even for certain strains, this led to the duplication of some genes and caused redundancies in many functions. These redundancies are only present at the DNA level because homologous genes between chromosomes and plasmids usually have different promoters and terminators, indicating that they are controlled by different regulatory elements at the transcriptional level.
Moreover, gene families that function as transcriptional regulators showed much greater abundance on plasmids, and they could be involved in the control of genes on plasmids. Data from transcriptomics and proteomics studies have indicated that genes on chromosomes are more active than those on plasmids [31,33]. Moreover, shared genes in the same cell have higher levels of transcription and translation on plasmids than on chromosomes. We suggest that genes on plasmids are more strictly controlled by regulators, which could neutralize the redundancies caused by homologous genes.

To survive in varied environments (soil, water, and animal hosts), members of the $B$. cereus group employ HGT to take up different types of genes that assist in adaptation and can integrate these genes into chromosomes or plasmids [21,34-36]. When a strain has existed in a steady environment for a long time, some essential genes may be integrated into the chromosome, whereas non-essential genes must be controlled more strictly or even lost. However, for the entire group, as the environment changes frequently, adaptive genes must be preserved on plasmids or chromosomes. This practice contributes to the survival of members of this group in different types of environments.

This study focused on the shared genes between plasmids and chromosomes, which provides somewhat incomplete evidence for the above conclusion. More analyses based on genome information and more laboratory experiments testing these deductions are needed in future work.

\section{Conclusion}

All of the plasmids were transferred frequently among members of the group and mediated numerous genetic exchange events among plasmids and between plasmids and chromosomes. For the entire group, most genes were located on both plasmids and chromosomes, with the copies on plasmids being more strictly controlled. We suggest that plasmids are vectors for redundant genes on chromosomes.

\section{Methods}

\section{Sequence collection}

The genome sequences of 104 plasmids ( 80 from GenBank and 24 from our group) were used in the analyses. The genome sizes of these 104 plasmids ranged from $\sim 2 \mathrm{~kb}$ to $\sim 566 \mathrm{~kb}$ (Additional file 4: Table S1). The sequences of 20 chromosomes (18 from GenBank and 2 from our group) were used for the shared gene analysis (Additional file 4: Table S2).

\section{Gene clustering}

Protein sequences longer than 50 amino acids from all chromosomes and plasmids were searched using BLASTP [37] with an all-against-all style and the default parameters. 
Protein sequences with identities and coverage greater than $70 \%$ were then clustered into families using the program OrthoMCL with an inflation value of 2 [38].

All the start positions and end positions of shared genes on a plasmid or a chromosome were compared against a series of uniform randomly distributed numbers with the same length as the positions to determine whether the positions showed a uniform random distribution on the plasmid and chromosome. All the analyses were conducted in $\mathrm{R}$ [39].

\section{COGs, base composition, codon usage and operon analysis}

To identify chromosomal core genes and chromosomal distributed genes, one gene per family was randomly extracted from chromosomal clusters derived from $B$. cereus strains whose complete genome sequences were available. Unique genes from each chromosome of the above strains were combined to form chromosomal unique genes. Moreover, the plasmid distributed genes consisted of one random gene per family together with all the unique genes from all the plasmids whose genome sequences were available.

For the COG analysis, we constructed a local COG database [40] and ran RPSBLAST [37] using the sequence sets described above as queries with an e-value cutoff of 0.001 . We focused on the top three hits from each alignment and counted each category for comparison using an in-house Perl script. The base composition was analyzed using G-language [41], and a CAI (codon adaptation index) analysis was performed using codonW software (version 1.4.4, http://codonw.sourceforge.net/).

The operons were predicted by ProOpDB [42]. To compare the promoters and terminators of shared genes between chromosomes and plasmids, we collected 200-bp upstream and 100-bp downstream sequences for each coding region of all these shared gene pairs. Then, we compared these sequences using BLAST.

\section{Phylogenetic tree construction}

Each of the 40 families of sequences of genes shared between plasmids and the chromosomal core set were used for phylogenetic tree construction. A maximum likelihood tree was generated by the PhyML software [43] with bootstrap support calculated from 100 replicates after each group of sequences was aligned by Muscle [44].

All the statistical analyses were performed using in-house Perl scripts and R 2.15.1 [39].

\section{Availability of supporting data}

The data sets supporting the results of this article are included within the article and the additional files.

\section{Additional files}

Additional file 1: Table S3. The 598 gene families shared by plasmids and chromosomes.

Additional file 2: Table S4. Transcriptional profiles of shared genes between plasmids and chromosome that have similar promoter and terminator sequences among the four time points.

Additional file 3: Figure S1. The proportions of each COG category for the chromosomal core gene set, chromosomal distributed gene set, chromosomal unique gene set, plasmids and chromosomal shared gene set as well as all the genes on plasmids.

Additional file 4: Table S1. Plasmids analyzed in this study. Table S2 Genomes used in this study.

\section{Competing interests}

The authors declare that they have no competing interests.

\section{Authors' contributions}

SM, JDH and ZJS designed the study with help from PDH and RLF; ZJS, GZY and CSY performed the analysis; and ZJS, JDH and SM wrote the manuscript. All the authors approved the final version of the manuscript.

\section{Acknowledgments}

This work was supported by grants from the National High Technology Research and Development Program (863) of China (2011AA10A203), the China 948 Program of Ministry of Agriculture (G25), and the National Natural Science Foundation of China (31170047 and 31171901).

Received: 2 August 2014 Accepted: 24 December 2014

Published online: 22 January 2015

\section{References}

1. Wiedenbeck J, Cohan FM. Origins of bacterial diversity through horizontal genetic transfer and adaptation to new ecological niches. FEMS Microbiol Rev. 2011;35(5):957-76

2. Thomas CM, Nielsen KM. Mechanisms of, and barriers to, horizontal gene transfer between bacteria. Nat Rev Microbiol. 2005;3(9):711-21.

3. Ding H, Hynes MF. Plasmid transfer systems in the rhizobia. Can J Microbiol. 2009;55(8):917-27.

4. Johnson TJ, Nolan LK. Pathogenomics of the virulence plasmids of Escherichia coli. Microbiol Mol Biol Rev. 2009:73(4):750-74.

5. del Solar G, Giraldo R, Ruiz-Echevarria MJ, Espinosa M, Diaz-Orejas R. Replication and control of circular bacterial plasmids. Microbiol Mol Biol Rev. 1998;62 (2):434-64.

6. Smillie C, Garcillan-Barcia MP, Francia MV, Rocha EP, de la Cruz F. Mobility of plasmids. Microbiol Mol Biol Rev. 2010;74(3):434-52.

7. Bennett PM. Plasmid encoded antibiotic resistance: acquisition and transfer of antibiotic resistance genes in bacteria. Br J Pharmacol. 2008;153 Suppl 1: S347-57.

8. Tamminen M, Virta M, Fani R, Fondi M. Large-scale analysis of plasmid relationships through gene-sharing networks. Mol Biol Evol. 2012;29 (4):1225-40.

9. Fondi M, Bacci G, Brilli M, Papaleo MC, Mengoni A, Vaneechoutte M, et al. Exploring the evolutionary dynamics of plasmids: the Acinetobacter pan-plasmidome. BMC Evol Biol. 2010;10:59.

10. Halary S, Leigh JW, Cheaib B, Lopez P, Bapteste E. Network analyses structure genetic diversity in independent genetic worlds. Proc Natl Acad Sci U S A. 2010;107(1):127-32.

11. Norris $V$, Merieau A. Plasmids as scribbling pads for operon formation and propagation. Res Microbiol. 2013;164(7):779-87.

12. Siguier $P$, Filee J, Chandler M. Insertion sequences in prokaryotic genomes. Curr Opin Microbiol. 2006;9(5):526-31.

13. Lee CA, Babic A, Grossman AD. Autonomous plasmid-like replication of a conjugative transposon. Mol Microbiol. 2010;75(2):268-79.

14. Novick RP, Christie GE, Penades JR. The phage-related chromosomal islands of Gram-positive bacteria. Nat Rev Microbiol. 2010;8(8):541-51.

15. Darmon E, Leach DR. Bacterial genome instability. Microbiol Mol Biol Rev. 2014;78(1):1-39. 
16. Wang $Y$, Peng D, Dong Z, Zhu L, Guo S, Sun M. Cloning and analysis of a large plasmid pBMB165 from Bacillus thuringiensis revealed a novel plasmid organization. PLoS One. 2013;8(12):e81746.

17. Bobay LM, Touchon M, Rocha EP. Pervasive domestication of defective prophages by bacteria. Proc Natl Acad Sci U S A. 2014;111(33):12127-32.

18. Bobay $L M$, Rocha $E P$, Touchon $M$. The adaptation of temperate bacteriophages to their host genomes. Mol Biol Evol. 2013;30(4):737-51.

19. Guglielmini J, Quintais L, Garcillan-Barcia MP, de la Cruz F, Rocha EP. The repertoire of ICE in prokaryotes underscores the unity, diversity, and ubiquity of conjugation. PLoS Genet. 2011;7(8):e1002222.

20. Zheng J, Peng D, Ruan L, Sun M. Evolution and dynamics of megaplasmids with genome sizes larger than $100 \mathrm{~kb}$ in the Bacillus cereus group. BMC Evol Biol. 2013;13:262.

21. Vilas-Boas GT, Peruca AP, Arantes OM. Biology and taxonomy of Bacillus cereus, Bacillus anthracis, and Bacillus thuringiensis. Can J Microbiol. 2007;53 (6):673-87.

22. Guinebretiere MH, Auger S, Galleron N, Contzen M, De Sarrau B, De Buyser $\mathrm{ML}$, et al. Bacillus cytotoxicus sp. nov. is a novel thermotolerant species of the Bacillus cereus Group occasionally associated with food poisoning. Int $J$ Syst Evol Microbiol. 2013;63(Pt 1):31-40.

23. Ehling-Schulz M, Fricker M, Grallert H, Rieck P, Wagner M, Scherer S. Cereulide synthetase gene cluster from emetic Bacillus cereus: structure and location on a mega virulence plasmid related to Bacillus anthracis toxin plasmid pXO1. BMC Microbiol. 2006;6:20.

24. Berry C, O'Neil S, Ben-Dov E, Jones AF, Murphy L, Quail MA, et al. Complete sequence and organization of pBtoxis, the toxin-coding plasmid of Bacillus thuringiensis subsp. israelensis. Appl Environ Microbiol. 2002;68(10):5082-95.

25. He J, Shao X, Zheng H, Li M, Wang J, Zhang Q, et al. Complete genome sequence of Bacillus thuringiensis mutant strain BMB171. J Bacteriol. 2010;192(15):4074-5.

26. He J, Wang J, Yin W, Shao X, Zheng H, Li M, et al. Complete genome sequence of Bacillus thuringiensis subsp. chinensis strain CT-43. J Bacteriol. 2011;193(13):3407-8.

27. Reyes-Ramirez A, Ibarra JE. Plasmid patterns of Bacillus thuringiensis type strains. Appl Environ Microbiol. 2008;74(1):125-9.

28. Liu XJ, Ruan LF, Lin XY, Zhao CM, Zhong CY, Sun M. Distribution of 2-kb miniplasmid pBMB2062 from Bacillus thuringiensis kurstaki YBT-1520 strain in Bacillus species. Ann Microbiol (Paris). 2013;63:1639-44.

29. Zhong C, Peng D, Ye W, Chai L, Qi J, Yu Z, et al. Determination of plasmid copy number reveals the total plasmid DNA amount is greater than the chromosomal DNA amount in Bacillus thuringiensis YBT-1520. PLoS One. 2011;6(1):e16025

30. Zheng J, Peng D, Song X, Ruan L, Mahillon J, Sun M. Differentiation of Bacillus anthracis, $B$. cereus, and $B$. thuringiensis on the basis of the $c s a B$ gene reflects host source. Appl Environ Microbiol. 2013;79(12):3860-3.

31. Wang J, Mei H, Zheng C, Qian H, Cui C, Fu Y, et al. The metabolic regulation of sporulation and parasporal crystal formation in Bacillus thuringiensis revealed by transcriptomics and proteomics. Mol Cell Proteomics. 2013;12 (5):1363-76.

32. Landeta C, Davalos A, Cevallos MA, Geiger O, Brom S, Romero D. Plasmids with a chromosome-like role in rhizobia. J Bacteriol. 2011;193(6):1317-26.

33. Wang J, Ai X, Mei H, Fu Y, Chen B, Yu Z, et al. High-throughput identification of promoters and screening of highly active promoter-5'-UTR DNA region with different characteristics from Bacillus thuringiensis. PLoS One. 2013;8(5): e62960.

34. Bottone EJ. Bacillus cereus, a volatile human pathogen. Clin Microbiol Rev. 2010;23(2):382-98.

35. Stenfors Arnesen LP, Fagerlund A, Granum PE. From soil to gut: Bacillus cereus and its food poisoning toxins. FEMS Microbiol Rev. 2008;32(4):579-606.

36. Mols M, Abee T. Bacillus cereus responses to acid stress. Environ Microbiol. 2011;13(11):2835-43.

37. Mount DW. Using the Basic Local Alignment Search Tool (BLAST). CSH Protoc. 2007;2007:pdb top17.

38. Li L, Stoeckert Jr CJ, Roos DS. OrthoMCL: identification of ortholog groups for eukaryotic genomes. Genome Res. 2003;13(9):2178-89.

39. R Core Team. R: A Language and environment for statistical computing In: R Foundation for Statistical Computing. Vienna, Austria; 2012 URL: http://www.R-project.org/.

40. Tatusov RL, Fedorova ND, Jackson JD, Jacobs AR, Kiryutin B, Koonin EV, et al. The COG database: an updated version includes eukaryotes. BMC Bioinformatics. 2003:4:41.
41. Arakawa K, Mori K, Ikeda K, Matsuzaki T, Kobayashi Y, Tomita M. G-language Genome Analysis Environment: a workbench for nucleotide sequence data mining. Bioinformatics. 2003;19(2):305-6.

42. Taboada B, Ciria R, Martinez-Guerrero CE, Merino E. ProOpDB: Prokaryotic Operon DataBase. Nucleic Acids Res. 2012;40(Database issue):D627-31.

43. Guindon S, Dufayard JF, Lefort V, Anisimova M, Hordijk W, Gascuel O. New algorithms and methods to estimate maximum-likelihood phylogenies: assessing the performance of PhyML 3.0. Syst Biol. 2010;59(3):307-21.

44. Edgar RC. MUSCLE: multiple sequence alignment with high accuracy and high throughput. Nucleic Acids Res. 2004;32(5):1792-7.

\section{Submit your next manuscript to BioMed Central and take full advantage of:}

- Convenient online submission

- Thorough peer review

- No space constraints or color figure charges

- Immediate publication on acceptance

- Inclusion in PubMed, CAS, Scopus and Google Scholar

- Research which is freely available for redistribution 